\title{
A rare complication of laparoscopic adjustable gastric band: acute large bowel obstruction
}

\begin{abstract}
With many thousands patients carrying laparoscopic adjustable gastric band (LAGB), longterm complications can appear emergently to any surgeon. We present a 64 year-old male who underwent placement of a laparoscopic adjustable gastric band (LAGB) years prior presented to the emergency department with concerning signs of an acute abdomen. Upon imaging review a large bowel obstruction at the level of the sigmoid colon, with ischemic changes, was discovered, caused by the patient's LAGB catheter. The patient was taken emergently for an exploratory laparotomy during which the LAGB catheter was removed, resulting in restored bowel perfusion. Bowel obstructions are infrequent complications of LAGB. However most commonly they are limited to the small bowel. LAGB catheters resulting in large bowel obstructions are an extremely rare finding. Our review of the literature indicates that this is the first reported cause of a sigmoid obstruction caused by a LAGB. This case brings into view potential complications LAGB that can confront not only bariatric surgeons, but general and acute care surgeons as well. This report illustrates LAGB danger to the colon, and suggests how to manage, perhaps leading to early, lifesaving intervention.
\end{abstract}

Keywords: large bowel obstruction, laparoscopic adjustable gastric band catheter, obesity, acute abdomen
Volume 10 Issue 6 - 2020

\author{
Ajay Menon, Lisa E Pedevillano, Melissa L \\ Gott,Timothy S Pilla, Gus J Slotman \\ Department of Surgery, Inspira Health Network, USA
}

Correspondence: Gus J Slotman, Inspira Health Network, I505 West Sherman Avenue Suite B,Vineland, NJ08360, USA, Tel 856-64I-8635, Fax 856-64I-8636, Email slotmag@ihn.org Received: November 21, 2020 | Published: December 09,
2020

\section{Introduction}

Laparoscopic adjustable gastric band (LAGB) previously was one of the most common surgical options for the management of morbid obesity, but its popularity waned. After peaking at $42.3 \%$ of bariatric surgery operations in $2008,{ }^{1}$ by 2018 the total LAGB cases were approximately $1.1 \%$ of all bariatric surgery procedures. ${ }^{2}$ Several hundred thousand patients currently live with LAGB in place. Gastric banding was first introduced in 1986 and the laparoscopic approach was implemented in 1990. Although this technique is relatively simpler and less morbid when compared to the other available surgical therapies for morbid obesity, it is not without complications. The most common complications include pouch enlargement, band slip, band erosion, and port-site infections. ${ }^{3}$ After review of the current literature, rare complications have been reported as well including intraluminal band migration and volvulus resulting in obstruction or perforations. ${ }^{4-7}$ These complications are often associated with a significant morbidity and result in the revision or removal of the gastric band itself. Studies have been conducted with respect to LAGB associated complications to determine incidence and outline management options, however these complications are exceedingly rare and studies are limited to case series or reports. ${ }^{8}$ Through this case presentation we aim to highlight a unique complication by the LAGB catheter which may be of utility to bariatric and acute care surgery teams in the management of LAGB associated complications.

\section{Case report}

A 64-year-old male with a past medical history of diabetes, morbid obesity (BMI 44), prior surgical history of LAGB placement unknown years before, resulting in a reported 100 pound weight loss, presented to the emergency department with three days of abdominal pain. He reported his pain worsened over that timeframe with associated nausea. He denied any bowel function in the form of flatus or bowel movement. He was not in acute distress. Abdominal exam was pertinent for diffuse tenderness with rebound and guarding. Lab values were pertinent for a leukocytosis of 19 , neutrophils of 92.7, hemoglobin of 16.8 , platelets 499 . Basic metabolic panel included glucose 315, BUN 22, Creatinine 1.2, Sodium 136, Potassium 3.7, Chloride 102, CO2 15, Anion gap 19, Lactate 4.1. His venous blood gas revealed a $\mathrm{pH} 7.182, \mathrm{pCO} 261.8, \mathrm{pO} 225$, bicarbonate 23.7, and base excess -5.6. A CT scan of the abdomen and pelvis identified a large bowel obstruction at the level of the mid sigmoid colon with the LAGB catheter looped along the sigmoid mesentery in the pelvic inlet, as depicted in Figures 1 and 2. After resuscitation with 2 liters of crystalloid the patient was transferred urgently to the operating room for exploratory laparotomy. Intra-operatively marked dilation of the entire intestinal tract and a large loop of sigmoid colon was found to be strangulated by several loops of the LAGB catheter that wrapped along the rectosigmoid mesentery. The large bowel was decompressed proximally and distally with needle catheter suction. The enterotomies were closed with a figure of eight 3-0 polysorb suture. The sigmoid colon appeared dark and hemorrhagic as well. After extensive lysis of adhesions the catheter was excised and trimmed flush with the LAGB reservoir at the upper abdomen. The sigmoid colon was observed as it regained a viable color after a few minutes. In addition the mesenteric pulse was palpable. The abdomen was closed with a running 0 Maxon suture and the patient tolerated the procedure well. Post-operatively the patient progressed uneventfully. His discharge was delayed due to newly diagnosed hypertension, however once optimized he was discharged on post-operative day 7 (Figures 1) (Figures 2). 


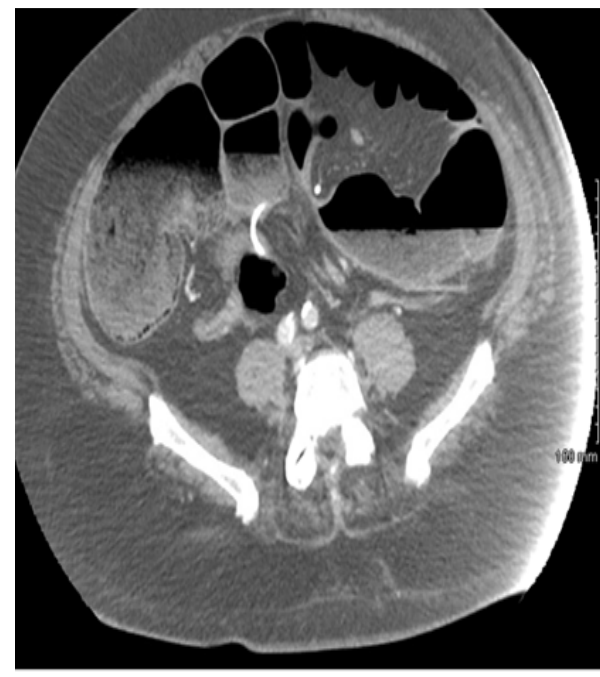

Figure I Axial Computed Tomography (CT) of Abdomen/Pelvis Demonstrating Acute Large Bowel Obstruction due to LAGB Catheter.

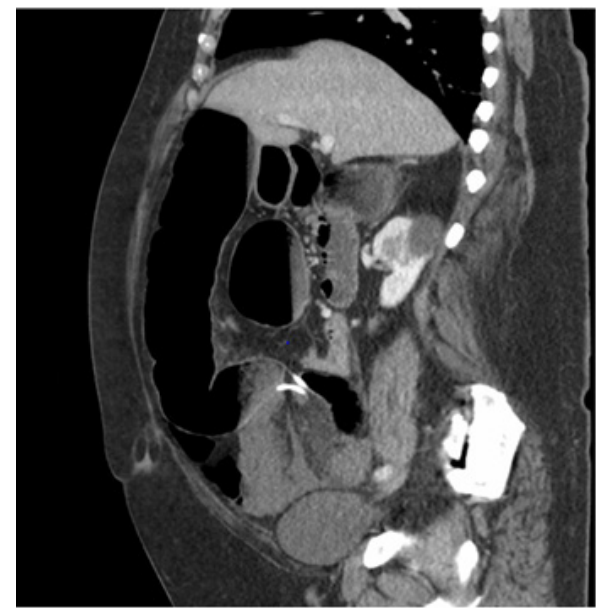

Figure 2 Sagittal CT of Acute Large Bowel Obstruction.

\section{Discussion}

Morbid obesity is a major medical illness globally with serious socioeconomic and medical consequences. With the advent of bariatric surgery there are now a variety of surgical corrective procedures to help patients with weight loss. LAGB now comprises only $1.1 \%$ of American bariatric procedures, hundreds of thousand of patients still carry the device, ${ }^{3}$ which still performs well in diet compliant patients. ${ }^{1,2}$ LAGB, although a restrictive procedure, is still with its share of complications, including band erosion and the potential for intraluminal migration, slippage, incisional hernias, port infection, or even small bowel obstruction. This case report presents a unique complication of LAGB surgery involving an acute sigmoid colon obstruction with constriction of the rectosigmoid mesentery secondary to a LAGB catheter. After review of the current literature, the majority of publications on bowel obstruction due to LAGB are limited to case reports describing small bowel obstructions caused by the device. Nonetheless, any bowel obstruction secondary to LAGB should be evaluated as a surgical emergency. It is important to understand that these patients may present with days to weeks of abdominal pain and, unlike the course of other causes of intestinal obstruction, will unlikely progress with conservative management as these obstructions are almost always closed-loop in nature. ${ }^{9}$
The length of the LAGB catheter is a clear causative factor of these bowel obstructions, both large and small. Typically, these tubing catheters are intentionally long and loose to aid with revision and removal in the event of band infection. As a result this tube redundancy is a risk factor for loops and resulting in long term complications such as bowel obstructions. ${ }^{9}{ }^{10}$ In our case we opted to excise the tube itself to prevent further complications. Other options may include fixating the tube to the anterior abdominal wall at the time of LAGB to minimize chances of redundancy.

An important aspect of this case is to illustrate the fact that these complications are in the realm of both bariatric and general surgeons. With the advent of bariatric surgery and its evolution a higher percentage of patients have undergone some form of prior foregut surgery that may present to the acute care surgeon. It is imperative for general surgeons to have an understanding of basic bariatric procedures and their complications as this can facilitate potentially life-saving interventions. Therefore, in LAGB patients presenting with signs and symptoms of an acute abdomen one must consider closed loop bowel obstructions on the differential diagnosis. There are some limitations to this study, mainly being that this study represents one isolated report of a large bowel obstruction from a LAGB catheter. Further research must be done regarding the optimal catheter length at the time of LAGB placement to minimize the risk of long term postoperative complications.

\section{Conclusion}

This case identifies a strangulation of the sigmoid colon along with its mesentery secondary to excessive tubing from LAGB, resulting in a closed loop obstruction. Caution must be taken when placing LAGB as surgeons must be cognizant regarding the amount of tubing left behind and its degree of redundancy. Further research must be done regarding catheter length and post-operative outcomes. Furthermore, all general surgeons must entertain the possibility of obstructions secondary to LAGB tubing in symptomatic patients.

\section{Acknowledgments}

None.

\section{Conflicts of interest}

We disclose no conflicts of interest.

\section{Funding}

None.

\section{References}

1. Buchwald H, DM Oien. Metabolic/bariatric surgery Worldwide 2008. Obes Surg. 2009;19(12):1605-1611.

2. Wayne J English, Eric J DeMaria, Matthew M Hutter, et al. American Society for Metabolic and Bariatric Surgery 2018 estimate of metabolic and bariatric procedures performed in the United States. Surg Obes Relat Dis. 2020;16(4):457-463.

3. Iyad Eid, Daniel W Birch, Arya M Sharm, et al. Complications associated with adjustable gastric banding for morbid obesity: a surgeon's guides. Can J Surg. 2011;54(1):61-66.

4. Bassam A. Unusual gastric band migration outcome: distal small bowel obstruction and coming out per-rectum. Pan Afr Med J. 2012;13:9.

5. Agahi A, R Harle. A serious but rare complication of laparoscopic adjustable gastric banding: bowel obstruction due to caecal volvulus. Obes Surg. 2009;19(8):197-200. 
6. Oppliger F, H Rios, L Manriquez. Migration and perforation of a gastric band in the small bowel. Cir Esp. 2015;93(9):601-603.

7. Oppliger F, G Wiedmaier, J Leon. Acute small bowel obstruction due to the connecting tube of a gastric band. Surg Obes Relat Dis. 2014;10(6):e7779.

8. Cherian PT, G Goussous, F Ashorii, et al. Band erosion after laparoscopic gastric banding: a retrospective analysis of 865 patients over 5 years. Surg Endosc. 2010;24(8):2031-2038.
9. Suter KJ, N Rajasagaram, P Nottle. Gastric band connection tube results in small bowel obstruction: an acute emergency. J Surg Case Rep. 2016;2016(5)

10. Osama H Hamed, Lashondria Simpson, Emanuele Lomenzo, et al. Internal hernia due to adjustable gastric band tubing: review of the literature and illustrative case video. Surg Endosc. 2013;27(11):4378-4382. 